II.

\title{
Zur Lehre von den römischen Korporationen.
}

\author{
Von \\ Theodor Mommsen.
}

Aus dem Nachlaß des Verfassers herausgegeben. ${ }^{\text {) }}$

Unter den mannigfaltigen Kreisen der römischen Rechtsordnung ist kaum ein zweiter für den Juristen so undurchsichtig und peinlich wie die Bestimmungen über die sogenannte juristische Person, genauer definiert über die Frage, inwiefern vermögensrechtlich neben der physischen Person noch anderweitige Rechtssubjekte auftreten. Hauptsächlich ist dies darin begründet, daß auf diesem Gebiet radikal verschiedene Rechtskreise sich berühren. Die gesetzlichen Beschränkungen des Assoziationsrechts und die Konsequenzen der dagegen vorkommenden Kontraventionen gehören von

1) Das Manuskript der beiden nachfolgenden, nach äußeren und inneren Kennzeichen vor nicht langer Zeit niedergeschriebenen Abhandlungen wurde durch gütige Vermittlung des Herrn Professors O. Hirschfeld von der Familie des Verfassers der Zeitschrift in freundlicher Weise zur Verfügung gestellt. Die Untersuchung über die römischen Korporationen ist wohl, wie namentlich in ihrem Schlußteil hervortritt, noch auf weitere Ausführung berechnet gewesen; einzelne Entwürfe, insbesondere die Rechtsstellung der Gottheiten betreffend, lagen auf losen Blättern bei, schienen aber zur Veröffentlichung nicht geeignet. Dagegen erachtete es die Redaktion im Einverständnis mit Professor Hirschfeld für unbedingt geboten, die Abhandlung selbst auch in ihrem gegenwärtigen Zustand zu veröffentlichen; obwohl Mommsen noch manches daran vervollständigt haben würde, stimmt sie doch in den Grundgedanken mit seinen sonst geäußerten Anschauungen so wohl überein, daß sie als seine letzte Meinung über die bez. Fragen angesehen werden kann. - Die Überschrift ist erst bei der Herausgabe beigefügt worden.

D. Bed.

Zeitschrift für Rechtsgeschichte. XXV. Rom. Abt. 
Rechts wegen dem Kriminalrecht an, greifen aber mit ihren Folgen in das Civilrecht ein. Wichtiger noch ist es, dab das von der physischen Person verschiedene vermögensrechtliche Subjekt zunächst und in gewissem Sinn allein der Staat ist und also diese Frage, theoretisch gefaßt, mehr dem Staatsrecht angehört als dem Privatrecht, in welchem sie uns vorwiegend entgegentritt. Es hat mich dies bestimmt, von diesem Gesichtspunkt aus in möglichster Kürze diese Lehre in der mir richtig erscheinenden Auffassung hier darzulegen. Dankbar bekenne ich mich dabei als Schuldner von Pernice, dessen Darstellung derselben vom civilrechtlichen Standpunkt aus (Labeo 1, 254-309) mir als Leitfaden und Anhalt gedient hat und auf den da, wo ich seinen Ausführungen einfach beitrete, hiermit verwiesen wird.

Die Rechtsstellung der Stadtgemeinde kann nicht erwogen werden ohne Rücksicht auf die Wandlungen, welchen dieser Begriff im Verlauf der Entwicklung des römischen Staats unterlegen hat. Derselbe ist seiner Anlage nach eine Konföderation selbständiger Stadtgemeinden, unter denen der populus Romanus wohl bevorrechtet, aber dem Souveränetätsbegriff nach nur einer von vielen ist, und welche in sich selbst Sondergemeinden nicht kennt; im Verlauf der Entwicklung aber wird einerseits die staatliche Selbständigkeit der föderierten Gemeinden mehr und mehr beschränkt, andererseits innerhalb der römischen Gemeinde namentlich für die in dieselbe aufgegangenen, einstmals föderierten Staaten, aber auch für andere örtlich separierte Gemeinschaften römischer Bürger eine hybride, der staatiichen Selbständigkeit genäherte Rechtsstellung entwickelt, welche schon von den römischen Juristen als das Stadtrecht im Staat, municipium, gefaßt wird. Dieses letztere, wie es sich wahrscheinlich nach der Aufnahme aller italischen Gemeinden in den italischen Bürgerverband rechtlich fixiert hatte, ist ein Bestandteil der römischen Rechtsordnung, wogegen die föderierten Gemeinden von Rechts wegen außerhalb derselben stehen, die latinischen Sonderrechte nur beiläufig, die der rechtlich oder faktisch autonomen peregrinischen fast nur gegensätzlich Berücksichtigung finden. Seit die Stadtrechte von Salpensa und Malaca uns einen Einblick in die latini- 
sche Stadtverfassung der früheren Kaiserzeit erschlossen haben, wird von den Juristen wohl verlangt werden dürfen, diese Verhältnisse auch da im Sinne zu behalten, wo unsere Überlieferung selbst von latinischen und peregrinischen Ordnungen nicht ausdrücklich spricht, zum Beispiel die civitates quae sub imperio populi Romani sunt (Ulp. 24, 28) nicht einfach mit den in den Digesten auftretenden Munizipien zu identifizieren.

Als leitendes Prinzip für die Behandlung des municipium civium Romanorum im Vermögensrecht möchte aufzustellen sein, daß es in der Rechtsfähigkeit dem populus Romanus gleichsteht, dagegen die Handlungsfähigkeit ihm nicht bloß in dem eminenten Umfang fehlt, wie er aus der souveränen Gewalt des populus Romanus resultiert, sondern in dieser Hinsicht die Stadtgemeinde dem des Vormunds entbehrenden Pupillen verglichen werden kann.

Fassen wir die geschichtliche Entwickelung dieser Verhältnisse ins Auge, so kann es keinem $\mathrm{Zweifel} \mathrm{unterliegen,}$ $\mathrm{da} \beta$ einerseits bei den föderierten Städten die römische Obergewalt erst später als auf dem eigentlich staatlichen Gebiet vermögensrechtliche Beschränkungen herbeigeführt hat, daß Praeneste wie Athen auch nach Eintritt der politischen Untertänigkeit sich innerhalb ihres Rechtskreises auf diesem Gebiet lange Zeit unabhängig behauptet haben; daß aber ebenso andererseits die römische Bürgergemeinde in älterer Zeit vermögensrechtlich so wenig Rechtssubjekt gewesen ist wie $\mathrm{zu}$ allen Zeiten die Kurie und die Tribus, wie dies die bekannte Tatsache, daß noch am Ende des sechsten Jahrhunderts der Stadt in den bestgestellten Bürgerkolonien die Gemeindebauten durch die römischen Censoren, und oft aus dem römischen Ärarium hergestellt wurden ${ }^{1}$ ), deutlich genug anzeigt. Aber für das uns überlieferte Civilrecht steht allerdings die Persönlichkeit der römischen Bürger-

1) Staatsrecht $2^{3}, 429.3,819$. Wenn hier gesagt ist, daß die Bürgerkolonie von Haus aus eigenes Vermögen gehabt haben kann, so ist auch das schon zu viel zugestanden; die Separierung, die allerdings im Wesen der Sache liegt, kann ursprünglich nur faktischer Art gewesen sein, ähnlich wie bei den Priesterkollegien. 
gemeinde außer $Z_{w}$ eifel ${ }^{1}$ ), und wenn vermutet werden darf, daß der einstmalige Besitz der Souveränetät, wie er als Regel bei dem Municipium angenommen werden kann, hierbei zugrunde liegt, und die Personalität der Stadt also vielmehr gelassen als beigelegt worden ist, so ist sie doch nicht minder auf die einstmals unselbständige colonia civium Romanorum erstreckt und in dieser Hinsicht zwischen beiden Kategorien kein Unterschied gemacht worden. ${ }^{2}$ ) Die vermögensrechtliche Gleichstellung des Gesammtstaats und der Stadt beweist auch die Terminologie: vermögensrechtlich aufgefaßt wird das Gemeindevermögen bezeichnet als res publica $^{3}$ ), die Gemeindekasse als aerarium. ${ }^{4}$ ) DaB streng genommen die Bürgergemeinde, als nicht souverän, nicht populus genannt werden darf, spricht sich allerdings in der won den römischen Juristen theoretisch aufgestellten ${ }^{5}$ ) und auch praktisch einzeln befolgten ${ }^{6}$ ) Regel aus, daß für publicum vielmehr commune gesetzt werden muß; aber sicher haben auch diese strengen Rechtslehrer der Stadtgemeinde nicht die

1) Florentinus Dig. 46,1.22: hereditas personae vice fungitur sicuti municipium et decuria et societas. $-{ }^{2}$ ) Frontinus p. 54, $22=85,28$ : "fundus Septicianus coloniae Augustae Concordiae": [haec inscriptio] videtur ad personam coloniae ipsius pertinere neque ullo modo abalienari posse a re publica. Andere Belege Pernice Labeo 1, 283. - ${ }^{3}$ ) Staatsrecht $2^{3}, 1084$ A. 1. C. I. L. V, p. 1195 . XIV, p. 578 und sonst. Wenn in poplicum dare von der Zahlung an die damals wahrscheinlich fồderierte Stadt Genua gebraucht wird (sent. Minuc. vom J.637 d. St. 1 . 25. 27), so steht weiterhin publicum bekanntlich ganz gewöhnlich vom städtischen Besitz (z. B. C. I. L. XIV a. a. O.) - ) Inschrift von Tergeste C. I. L. V, 532: aerarium nostrum ditavit. In den munizipalen Inschriften begegnen quaestor aerarii und quaestor pecuniae publicae häufig, quaestor arcae selten: quaestor aerar(ius) arcae publicae (Anagnia C. X, 5928), quaestor arcae r(ei) p(ublicae) Nepesinor(um) (Wilmanns 2086), quaestor arcae (Eburum C. X, 451). - s) Ulpian Dig. 50, 16, 15. Papinian

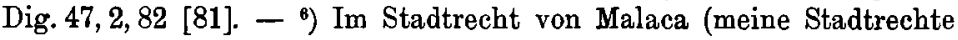
S. 457) steht meistens communis für publicus, doch findet sich einzeln auch letzteres. In den severischen Säkularakten scheint dies sogar angewandt zu sein auf die Kasse der Stadt Rom (eph. epigr. 8, p. 280 1.23 cf. p. 297).-Diese Dokumente gehen von der römischen Regierung aus; die Städte selbst fügten sich der hierin enthaltenen Degradierung nicht.

Anm. der Red. zu Note 2: [haec inscriptio] im Ms. eingeklammert; die Klammer, welche in der Lachmannschen Ausgabe fehlt, deutet wohl an, daß der Verf. die Worte für ein Glossem hielt. 
Persönlichkeit absprechen, sondern sie nur als nicht auf dem gleichen Fundament mit derjenigen des Staats stehende, vielmehr exzeptionell zugelassene charakterisieren wollen.

Neben der anerkannten Rechtsfähigkeit steht die Handlungsunfähigkeit. Anerkannt und motiviert wird dieselbe namentlich von Ulpian in der bekannten Begründung der Unzulässigkeit der Erbeinsetzung der Stadtgemeinde (22, 5): nec municipia nec municipes heredes institui possunt, quoniam incertum corpus est, ut neque cernere universi neque pro herede gerere possint ${ }^{1}$ ), ut heredes fiant. Die viel umstrittenen Worte sind wohl so zu interpretieren, daß die Stadt zwar ein Rechtssubjekt ist (corpus), aber die sie bildenden physischen Personen nicht mit rechtlicher Sicherheit determiniert werden können, es also auch nicht möglich ist, den gleichmäßigen Willen aller Zugehörigen zu konstatieren. Daß dies richtig ist, eine Konstatierung der sämtlichen Gemeindebürger für einen gegebenen Zeitpunkt nicht in der Weise möglich ist wie zum Beispiel bei der Apparitorendekurie oder dem Priesterkollegium ${ }^{2}$ ), kann nicht bestritten, wohl aber hinzugesetzt werden, daß, selbst wenn man die zustimmenden Bürger als die vollzählige Bürgerschaft ansehen wollte, den gleichartigen Willenserklärungen mehrerer Personen das Moment der rechtlichen Einheit noch abgehen würde. ${ }^{3}$ ) Man

1) Es ist wohl possunt in possint zu ändern, nicht ut in et. Vorher ist wohl municipium zu setzen statt municipia. - $\left.{ }^{2}\right)$ Paulus Dig. 34, 5, 20: (collegio) cui (coive) non licet si legetur, non valebit, nisi singulis legetur: hi enim non quasi collegium, sed quasi certi homines admittentur ad legatum. - ${ }^{3}$ ) So muß die Stelle gefaßt werden, wenn man et ... possunt liest; aber mir erscheint dies für die römische Argumentation alløu spekulativ. Auch wenn Ulpian Dig. 41, 2, 1, 22 der Bürgerschaft die Möglichkeit abspricht, zu besitzen, quia universi consentive non possunt, und das gleiche wiederholt in Beziehung auf die erbrechtliche bonon'um possessio (Dig. $38,3,1,1$ ), ist dasselbe gemeint: es läßt sich immer nur eine größere Zahl von Bürgern konstatieren, nicht aber der Nachweis erbringen, daß es andere Bürger zur Zeit nicht gibt. - Wenn Pernice (Labeo 1, 286) einwendet, daß Ulpians Begründung nur zur Incapacität führt, nicht zur Erbunfähigkeit, so ist dagegen zu erwidern, da $\beta$ die Incapacität der augustischen Gesetzgebung in der Tat nichts ist, als eine durch die Zulassung nachträglicher Beseitigung des Hindernisses bedingte Capacität, aber die absolute Incapacität, wie sie hiernach gegeben ist, mit der Erbunfähigkeit zusammenfällt. 
wende dagegen nicht ein, daß im öffentlichen Recht die nach rechter Ladung erschienenen Bürger als Bürgerschaft gelten und teils durch die Majoritätsordnung, teils in späterer Zeit durch das decurionale Repräsentativsystem die Willensfindung der Gemeinde weiter vereinfacht wird; denn nirgends ist ausgesprochen, daß diese Sätze auf dem vermögensrechtlichen Gebiet zur Anwendung kommen, die Comitien oder späterhin der Ordo in Beziehung auf die Bürgerschaft eine ähnliche Stellung einnehmen wie sie dem Vormund in betreff des Mündels zusteht. ${ }^{1}$ ) Daß bei dieser theoretischen Strenge die Absicht zugrunde liegt, die Stadtgemeinde auch vermögensrechtlich zu vinkulieren, ist glaublich genug, um so mehr, wenn man sich der ursprünglichen absoluten Unselbständigkeit der Bürgergemeinde erinnert; aber dies Ziel wurde nicht durch direkte Repression erreicht, sondern durch Anwendung der rechtlichen Konsequenz.

Es bleibt übrig, die uns vorliegenden Einzelbestimmungen darauf zu prüfen, ob sie diesen Prinzipien entsprechen, das heift, entweder sich als Ausflub derselben betrachten lassen, oder, wo sie denselben widerstreiten, auf römisches Spezialgesetz zurückgeführt werden können.

Daß die ordentliche Vermögensverwaltung, Kauf und Verkauf, Verpachtung, Arbeitsmiete und so weiter, wesentlich in demjenigen Umfang, wie sie in den römischen Ämtern der Censur und der Quästur enthalten ist, von jeder Bürgergemeinde ausgeübt wird, bedarf keines Beweises; selbst in den Bestand des Gemeindevermögens tief eingreifende $\mathrm{Maß}$ regeln, wie der Verkauf der der Gemeinde gehörigen Grundstücke, stehen in der Kompetenz derselben. ${ }^{2}$ ) Hierfür aber

1) Daß der Ordo die Gemeinde vertritt, wird wohl öfter ausgesprochen, z. B. bei Ulpian Dig. 3, 4, 3: mulli permittitur nomine civitatis vel curiae experivi nisi ei cui lex permittit aut lege cessante ordo dedit, cum duae partes adessent aut amplius quam duae. Aber daraus, daß für eine der Stadt von Rechts wegen zustehende Klage, wenn diese nicht nach ihrem Stadtrecht einem ihrer Magistrate zusteht, der Ordo den Syndikus ernennt, folgt nichts für den Umfang des klagbaren Rechts. [Gemeint ist jedenfalls "Erwerb des klagbaren R." (Anm. d. Red.)] — ${ }^{2}$ ) Siculus p. 158,5: has terras (die dem Fluß anliegenden und 
gibt genügende Deckung die Sanktion einer jeden Munizipalordnung durch die römische Gesetzgebung, welche, indem sie die Bestellung der betreffenden Gemeindebeamten anordnet, ihnen zugleich die mit dem Amt verknüpfte Kompetenz überweist. Darin liegt allerdings auch eine Schranke: wenn der betreffende Beamte seine Kompetenz überschreitet, zum Beispiel ein für die Gemeinde aufgenommenes Darlehen nicht in die öffentliche Kasse bringt oder zu ihrem Nutzen verwendet, so haftet dieselbe dem Darleiher nicht ${ }^{1}$ ); denn die Vertretung ist nicht formaler Art wie die vormundschaftliche, sondern nach publizistischer Weise tatsächlich zu fassen. Es kann sich also nur handeln um die nach römischer Auffassung außerhalb der Kompetenz der ordentlichen Gemeindebeamten liegenden vermögensrechtlichen Akte.

Dahin gehören vor allen Dingen die Freilassungen als Liberalitätsakte. In Beziehung auf diese aber steht es fest, daß durch einen im Jahre $129 \mathrm{n}$. Chr. auf die Provinzen erstrecktes Gesetz den Munizipien das Recht eingeräumt ward, ihre Sklaven zu manumittieren und ist es mindestens wahrscheinlich, daß dies ein Volksbeschluß der republikanischen Zeit ist ${ }^{2}$ ); es ist nicht wahrscheinlich, daß die Bürgergemeinden lange Zeit ohne diese praktisch unentbehrliche Befugnis die Verwaltung haben führen können. Wahrschein-

mit Rücksicht auf dessen Austreten von der Adsignation ausgeschlossenen Bodenstücke, vgl. p. 52,11) Pisaurenses publice vendiderunt, quas credendum est proximos quosque contingentes eas emisse vicinos.

1) Ulpian 12, 1,21: civitas mutui datione obligari potest, si ad utilitatem eius pecuniae versae sunt: alioquin ipsi soli qui contraxerunt, non civitas tenebuntur. -- 2) Diocletian cod. Iust. 7, 9, 3 fordert für die Gültigkeit der Freilassung von seiten einer Stadtgemeinde, daß sie erfolgt sei secundum legem uetti (oder uetiti)lybici, cuius potestatem senatusconsulto Iuventio Celso iterum et Neratio Marcello coss. facta ad provincias porrectam constitit. Daß in der Korruptel nicht der Name des Rogators im Genetiv stehen kann, leuchtet ein; daß mein Vorschlag veteris rei $p$. wenigstens der Sache nach das Richtige trifft, stellen die libertini municipio manumissi Varros (de l. lat. 8, 83) außer Zweifel. Die prinzipielle Rechtfertigung des Gewohnbeitsrechts bei Julian (Dig. $1,3,32)$ identifiziert den Willen sämtlicher Bürger mit dem der Bürgerschaft und es wird geraten sein, auch hier dieselbe, wenngleich inkorrekte Auffassung zugrunde zu legen. 
lich ist dabei zugleich das patronatische Intestaterbrecht auf die Stadtgemeinde übertragen worden; es ist dies ein ebenfalls praktisch unentbehrliches Korollar der Manumission und sowohl das Schweigen unserer Rechtsquellen über dies Recht der Stadtgemeinde auf die Intestatsuccession, sowie nicht minder das ihren Freigelassenen gegenüber ausnahmsweise eingeräumte Recht der Erbfolge nach dem Testament ${ }^{1}$ ) scheinen zu fordern, daß ihnen die Intestaterbfolge schon früh gestattet worden ist.

Daß die Stadtgemeinde, abgesehen von dem eben erwähnten Fall, nicht testamentarisch zum Erben eingesetzt werden kann, ist bereits erörtert worden; auch für den Erbschaftsantritt mangelt es an einer ordnungsmäßig zuständigen Behörde. Daß dabei die Absicht mit obwaltete, die Stadtgemeinde zu vinkulieren, ist bereits hervorgehoben worden; weniger indes wohl aus Rivalitätsgründen, für die kein rechter Raum ist, als weil der Erbschaftsantritt wegen der möglichen Insolvenz gefährlich werden kann. Das Universalfideikommiß unterliegt nach der rechtlichen Konsequenz der gleichen Behandlung; indes ist späterhin durch Senatsbeschluß die Annahme desselben gestattet worden. ${ }^{2}$ )

Dagegen ist nicht abzusehen, aus welchen theoretischen oder praktischen Gründen der Stadt die Erwerbung eines Legats hätte verwehrt werden können, da der Anfall weder für die Vindikation, noch für die Damnation eines besonderen Willensakts bedurfte, auch schon aus augusteischer Zeit ein Einzelbeleg dafür vorliegt. ${ }^{3}$ ) Nur wird daran zu erinnern sein, daß zwischen der Stadtgemeinde und dem Testator Rechtsgemeinschaft bestehen mußte, die föderierte oder latinische Stadt von einem römischen Bürger kein Legat empfangen durfte; ja es ist wohl denkbar, daß die Rechtsgemeinschaft auf den munizipalen Kreis eingeschränkt war, der Municeps nur seiner eigenen Gemeinde ein Legat zuwenden durfte. Wenn es bei Ulpian ${ }^{4}$ ) heißt: civitatibus omnibus, quae sub imperio populi Romani sunt, legari potest, idque a divo Nerva introductum postea a senatu auctore Hadriano diligentius constitutum est, so zeigt schon die Fassung, daß

1) Ulpian 22,5 , Dig. $\left.38,3,1,1 .-{ }^{2}\right)$ Ulpian a. a. O. $-{ }^{3}$ ) C. I. L. X. 5056. - 4) $24,28$. 
Nerva dies Recht nur verallgemeinert hat, offenbar in dem Sinn, daß jeder römische Bürger oder vielmehr jeder römische Reichsgenosse jeder zum römischen Reich gehörigen Stadtgemeinde ein Legat zuwenden konnte.

Wenn der Stadtgemeinde die Fähigkeit zu besitzen abgesprochen wird, so zeigt die Ausführung, daß dabei an die im allgemeinen Gebrauch stehenden Bodenstücke und Gebäude gedacht ist, bei welchen allerdings Vertretung nicht obwaltet und nur gefragt werden kann und gefragt worden ist, ob die Gemeindesklaven, welchen die Obhut derselben anvertraut ist, den Besitz derselben begründen, wobei freilich wieder die Gegenfrage gestellt werden kann, ob die Gemeinde diese Sklaven selbst besitze. ${ }^{1}$ ) Soweit dagegen die ordnungsmäßige Gemeindeverwaltung reicht, wird der Besitz, zum Beispiel der in der Gemeindekasse bewahrten Gelder und Wertsachen, der verpachteten Gemeindeländereien, insofern anerkannt, als die Bürgerschaft hier nicht 'durch sich selbst' besitzt.

Besondere Schwierigkeit macht die Schenkung. In unseren Rechtsquellen wird eingehend gehandelt sowohl von der Aufstellung von Bildsäulen in der Absicht, dieselben der Stadt bleibend zuzuwenden ${ }^{2}$ ), wie auch von dem Schenkungsversprechen sei es eines der Stadt zum Nutzen gereichenden Gebäudes, sei es einer Geldsumme. In beiden Fällen ist der

1) Paulus Dig. 41, 2, 1, 22: municipes per se nihil possidere possunt, quia universi consentire non possunt: forum autem et basilicam hisque similia non possident, sed promiscue his utuntur. sed Nerva filius ait per servum quae peculiariter adquisierint, et possidere et usucapere posse: sed quidam contra putant, quoniam ipsos servos non possident. Die Argumentation scheint verkürzt, da es sich nicht um das Besitzen allgemein, sondern um das Besitzen per se handelt. Nerva wird bemerkt haben, daß an dem Pekulium des servus publicus, zum Beispiel des arcarius, der Gemeinde der Besitz zweifellos zustehe und sie in ähnlicher Weise auch durch den servus custos basilicae sowohl dessen Pekulium als die Basilika besitzen könne, wogegen aber eingewendet ward, daß der Besitz des Sklaven selbst in dem zweiten Fall in Zweifel gezogen werden könne. Daß die Stadt per alium besitzen könne, wird in den zwar wohl interpolierten, aber sachlich zutreffenden Worten Dig. 38, 3, 1, 1 ausdrücklich gesagt. Auch Dig. 10, 4, 7, 3. 50,12,3,1 werden sie anerkannt als fähig, zu besitzen. $-{ }^{2}$ ) Die Rechtsgelehrten differieren insofern, als die strengere Ansicht bei der Aufstellung ea mente ut municipii esset (Dig. 44, 1, 23) der Stadt das Eigentum abspricht (Dig. 
leitende Gedanke offenbar der, daß diese Akte streng genommen notwendig einseitig $\left.{ }^{1}\right)$ und nicht vollgültig sind, sodaß nicht bloß von dem Akzept nirgends die Rede ist, sondern selbst die Erfüllung des Schenkungsversprechens das Geschäft nicht vollgültig macht. ${ }^{2}$ ) Selbstverständlich werden daneben durch außerordentliche Rechtshilfe die Zuwendungen wenn auch in gewissen Schranken aufrechterhalten. Die theoretische Begründung der Rechtsungültigkeit der Schenkung an die Gemeinde, einerlei ob deren Ratsversammlung oder deren Beamte sich damit einverstanden erklärt hatten oder nicht, kann nur darin gefunden werden, $\mathrm{da} B$ im Stadtrecht dieser außerordentliche Fall nicht vorgesehen war und es also für deren Akzept nicht, wie für die Verpachtung oder die Dingung, eine kompetente Behörde gab. Tatsächlich bestimmend für diese derjenigen des Legats völlig entgegengesetzte Behandlung der Schenkung an die Gemeinde ist offenbar die Rücksicht auf den Ambitus und überhaupt den ungebührlichen Einfluß gewesen, den der reiche Bürger auf diesem Wege sich verschaffen konnte; wer sich an die analogen Verhältnisse in Rom und an die in den Inschriften wie der pompejanischen Mauern so auch zahlreicher anderer Ortschaften erinnert, der wird die prinzipielle Repression solcher Schenkungen als völlig gerechtfertigt bezeichnen müssen. Die souveräne Oberherrschaft, welche in Italien der Senat, in den Provinzen die Statthalter und schließlich im ganzen Reiche die Kaiser über die Bürgergemeinden ausübten, gab wenigstens im allgemeinen das Mittel an die Hand, zu weit gehende Konsequenzen dieser Repression abzuschneiden und unter Beseitigung der schädlichen Freigebigkeit den Gemeinden die wahrhaft gemeinnützige Wohltätigkeit ihrer hervorragen-

41, 1,41: civium non esse), andere die Bildsäule betrachten als quasi publicata (Dig. 43, 24, 11, 1), andere geradezu als publica (Dig. 42, 5, 29); darin stimmen sie überein, daß sie tatsä.chlich der Gemeinde verbleibt und es weder dem Aufsteller, noch, wenn er in Konkurs gerät, dem Massenkäufer (Dig. 42, 5, 29) gestattet ist, sie zurückzunehmen.

1) Ulpian Dig. 50,12,3 pr.: pactum est duorum consensus atque conventio, pollicitatio vero offerentis solius promissum. - ${ }^{2}$ ) Ulpian Dig. $50,12,3,1$ : si quis quam ex pollicitatione tradiderat rem municipibus vindicare velit, repellendus est a petitione, aequissimum est enim huiusmodi voluntates in civitates collatas paenitentia non revocari. 
den Bürger zugute kommen zu lassen. Auf diesem Wege hat sich das Institut der Pollicitation entwickelt: unter Beseitigung der Klagbarkeit des Schenkungsvertrags wurde dem einseitigen Schenkungsversprechen an die Gemeinde Klagbarkeit beigelegt, wenn dasselbe entweder auf einem gerechtfertigten Motiv beruhte oder zu teilweiser Ausführung gelangt war. Das also fast unvermeidliche Willkürregiment hat freilich, wie begreiflich, den munizipalen Ambitus wohl einigermaßen beschnitten, aber sein Weiterwuchern keineswegs gehindert.

Dies gilt von der Rechtsstellung der römischen Bürgergemeinde. Der latinischen und der föderierten Stadt wird umgekehrt trotz des Reichsverbandes zunächst auf dem vermögensrechtlichen Gebiet neben der Rechtsfähigkeit die durch die Souveränetät gegebene volle Handlungsfähigkeit wesentlich in demselben Umfange zugestanden haben wie der römischen Gemeinde selbst; allerdings hätte König Attalus für sein pergamenisches Reich sicher nicht die Stadt Praeneste oder die Stadt Athen zum Erben einsetzen können, wie er dies für Rom tat. Spuren der einstmaligen Selbständigkeit der verbündeten Stadt zeigen noch die für Spanien in der Zeit der Flavier erlassenen Stadtrechte; aber es sind nur Spuren: daß selbst terminologisch das Gemeindevermögen von Malaca nicht res publica, sondern res communis genannt wird, ist ein deutlicher Hinweis [wie] auf die reale Gleichstellung dieser Städte mit denen der eigentlichen Bürgerschaft, [so auf die formale Differenzierung.] ${ }^{1}$ ) Den Prozeß der Umwandlung dieser alten Gemeindeunabhängigkeit in einfache Untertanenschaft im einzelnen zu verfolgen, würde von hohem geschichtlichen wie systematischen Interesse sein; indes fehlen uns dafür die Beweisstücke gänzlich.

Von dem populus Romanus mit unbeschränkter Rechtsund nicht minder unbeschränkter Handlungsfähigkeit sowie von dem ihm nachgebildeten municipium civium Romanorum geht die weitere Entwicklung der juristischen Person bei den Römern aus; sie ist, wenn wir von der aus dem Privatrecht

1) Die eingeklammerten Worte fehlen in Ms. und sind als durch den Zusammenhang gefordert eingesetzt worden (Aum. d. Red.). 
hervorgegangenen staatlichen societas absehen, wesentlich die mehr oder minder vollkommene privatrechtliche Verselbständigung eines Organs des Staates oder der Stadt.

Die Unteilbarkeit der Person, die Grundlage des Vermögensrechtes überhaupt, wird wie für das Individuum, so auch im Privatrecht für den Staat und die Stadt festgehalten. Es gilt dies sowohl in staatlicher wie in sakraler Beziehung.

Das Vermögensrecht kennt nur den Staat als solchen, nicht Bürgerschaft oder Senat, nicht die einzelnen Magistraturen, nicht den Ritterstand, nicht die Kurie oder die Tribus oder einen anderen Volksteil. Wie umfassend die Verwaltung dazu führt, insbesondere die Staatsdienstadministration zu teilen, wie häufig und wichtig die Verrechnungen zwischen den verschiedenen öffentlichen Kassen auch sind, insbesondere zwischen der Hauptkasse in Rom und denen der auswärts fungierenden Magistrate, der Träger der Rechte ist und bleibt ungeteilt der Populus. Ebenso wird in der Kaiserzeit das Kaisergut behandelt: administrativ gibt es zahllose kaiserliche Kassen und Verwaltungen; aber im Rechtssinn ist der Träger für alle der Fiskus und verhalten sich die Einzelkassen zueinander wie die zweier demselben Herrn gehöriger Landgüter. Das gleiche gilt in ihrem Kreise von der Stadt; auch hier ist privatrechtlich diese allein und ungeteilt der Träger ihrer Vermögensrechte.

Hinsichtlich der Götter gilt nicht ganz das gleiche. Was dem Staat und was der einzelnen dem Staat angehörigen Gottheit gehört, wird unterschieden. Aber vermögensrechtlich ist dennoch die staatlich anerkannte Gottheit keineswegs selbständiges Rechtssubjekt, ihr Gut nicht in dem Sinne aus dem staatlichen ausgeschieden wie das Privatgut. Man kann im wesentlichen das Verhältnis des Staates zu dem Tempelgut dahin präzisieren, daß dasselbe durch eine als dauernd gedachte religiöse Zweckbestimmung aus dem Staatsgut ausgeschieden ist, aber den Privaten gegenüber immer noch fortfährt, als Staatsgut zu gelten. Der Tempel steht rechtlich nicht viel anders wie der Markt und die Straße: vermögensrechtlich hat an jenem wie an dieser das Eigentum die Gemeinde, und die dauernde Zweckbestimmung dort für den Kultus, hier für den Verkehr entzieht jenen wie diese der ökono- 
mischen Verwertung. Allenfalls könnte man hinzusetzen, daß die persönlich gedachten Götter ihr Gut sozusagen als Pekulium innehaben, was bei dem Markt und der Straße nicht zutrifft. Über den Boden des verlegten Tempels disponiert der Staat genau ebenso wie über den Boden der verlegten Straße und das Rechtsgeschäft eines Privaten mit einem Tempel wird nicht nach dem Privatrecht, sondern gleich dem Rechtsgeschäft zwischen dem Privaten und der Gemeinde behandelt. Wenn in den Formen der Dedikation und der Konsekration der Gottheit eine Zuwendung bei Lebzeiten gemacht werden kann, und wenigstens dem kapitolinischen Jupiter, späterhin auch einzelnen anderen Gottheiten selbst die Erbfähigkeit beigelegt worden ist, so kann dies nur in dem Sinne verstanden werden, daß das also der Gottheit gegebene Vermögensstück dem Vermögen der Gemeinde zufällt unter der Verpflichtung, dasselbe gleich dem Tempelgut der betreffenden Gottheit zu behandeln.

Aber diesem Prinzip tut die Kollegialität nicht von Haus aus, aber im Laufe der Zeit einen großen Abbruch. Unter der Kollegialität versteht man den von seiten der Gemeinde einer gewissen Anzahl von Bürgern erteilten Sammtauftrag, im Gegensatz einerseits zu dem Einzelmandat, andererseits zu den allgemeinen Bürgerleistungen. Gleichgültig ist es dabei, ob diese Vergesellschaftung befristet ist, wie dies bei den Magistratskollegien der Fall ist, oder Eintritt und Abgang der Genossen in der Weise geordnet sind, daß die Gesellschaft dabei fortbesteht; gleichgültig ferner, ob die Vergesellschaftung herbeigeführt wird durch Volkswahl oder durch magistratische Berufung, wie bei den Apparitoren, oder durch Kooptation. DaB der Begriff durchaus ein publizistischer ist, der Auftrag, den die Genossen erhalten, gedacht ist als Gemeindemandat, die auf bloßer Privatwillkür beruhende Vergesellschaftung wohl eine sodalitas, aber kein conlegium begründet, zeigt sich unverkennbar, wenn man die ursprünglichen Kategorien in das Auge faßt und sich nicht durch die verwirrende Mannigfaltigkeit der Institutionen der Kaiserzeit den Blick trüben läßt.

An die Magistratskollegien genügt es zu erinnern. Daß auch die intervallierenden und die außerordentlichen Magis- 
traturen als collegia gefaßt werden, beweist, daß die Perpetuität zum Begriff des Kollegiums keineswegs gehört.

DaB die Apparitoren ebenfalls in diesen Kreis gehören, leuchtet ein; wenn ihre Genossenschaften technisch nicht collegia heißen, sondern decuriae, so ist dies vielleicht Zufall, eher aber wohl daraus zu erklären, daß sie hier ihre Dienstlöhnung empfingen, was von den übrigen Kollegien nicht gilt, und sie deshalb wohl von der vornehmeren Benennung ausgeschlossen wurden.

Über die numanischen Handwerkerinnungen sind wir so wenig unterrichtet, daß sie für den Begriff des Kollegium wenig in Betracht kommen. Daß sie aber nicht minder wie die Dekurien der Apparitoren von Staatswegen konstituiert wurden und staatliche Bedürfnisse befriedigten, folgt einfach daraus, daß sie bezeichnet werden als gebildet vom König Numa. Wahrscheinlich hängt auch ihr frühes Verschwinden damit zusammen, daß die Bedürfnisse, für die sie geordnet waren, in der Großstadt ohne Zutun der Regierung ihre Befriedigung fanden.

Der publizistische Zweck des Pontifikats und der übrigen rechtweisenden sakralen Kollegien ist evident.

Für die sehr zahlreichen Kollegien, welche dem Kultus einer bestimmten Gottheit gewidmet waren, wie zum Beispiel das der Arvalen, ist paradigmatisch der älteste darüber in den Annalen erhaltene Bericht: diesem zufolge wird wenige Jahre nach Vertreibung der Könige der Tempel des Mercurius geweiht und nach Beschluß des Senats und der Bürgerschaft der eine der Konsuln beauftragt, zur Versehung des dem neuen Gott gebührenden Dienstes ein collegium mercatorum einzurichten, welche Gesellschaft als Mercuriales späterhin mehrfach erwähnt wird. Es war dies nicht die einzige Form, um einen Tempeldienst zu ordnen; aber sicher ist sehr häufig bei der Einrichtung neuer sacra publica populi Romani in dieser Weise verfahren worden. Daß religiöse Vergesellschaftungen, welche im gleichen Fall nach dem in den Zwölftafeln anerkannten freien Assoziationsrecht im Privatwege sich bildeten, nicht als collegia galten, sondern als sodalitates, zeigen die bekannten Vorgänge nach der Aufnahme des Kultus der phrygischen Göttermutter im 
hannibalischen Kriege. Wahrscheinlich aber haben eben Assoziationen wie diese und wie die den Bacchuskult betreffenden zu der Denaturierung des publizistischen Begriffs den Anstoß gegeben: es lag ziemlich nahe, die Zulassung oder auch nur die Duldung eines neuen Kultus dahin zu interpretieren, daB darin zugleich eine generelle Gestattung der dafür sich bildenden religiösen Assoziationen enthalten sei, das heißt die erlaubten sodalitates als collegia aufzufassen. Wie eng die Bildung der Kollegien sich an die anerkannten öffentlichen Kulte anschließt, zeigt die Anknüpfung der stadtrömischen Kollegienbildung in der Revolutionszeit an die Kompitalien: der Larenkult eines jeden Kompitum war an sich gestattet, und die Benutzung dieser rechtlichen Grundlage ersparte der römischen Polizei das Einschreiten gegen die Organisierung des Gesindels.

Daß auch in der Kaiserzeit keine prinzipielle Änderung eintrat, ist in hohem Grad wahrscheinlich. Generelle Gestattung, wie sie zum Beispiel den collegia funeraticia zuteil ward, ist dafür nur eine Bestätigung. Daß auch innerhalb der Sklavenschaft collegia begegnen, kann ebensowenig dagegen geltend gemacht werden; es gilt von diesen Sklavengesellschaften, was von den Sklavenmagistraturen; Unfreie können, solange sie als solche auftreten, nicht der Usurpation mangelnden Rechts beschuldigt werden. Sicher hat auch oft genug eine Gesellschaft in dieser Zeit sich mißbräuchlich den vornehmeren Namen beigelegt; aber die Häufigkeit der collegia illicita ändert an der Rechtsordnung nichts. Daß die in der späteren republikanischen und in der Kaiserzeit in weitem Umfang auftretenden Beschränkungen des Assoziationsrechts in der Form der Kollegienverbote auftreten, erklärt sich ohne Zweifel daraus, daß, wie oben bemerkt, die unerlaubte Assoziation regelmäßig unter religiösen Formen sich versteckte. Hinsichtlich der munizipalen Kollegien spricht das eminente Hervortreten der mit dem Löschdienst verknüpften der fabri, centonarii, dendrophori für die Entstehung derselben durch öffentliche Autorität; noch entschiedener die Tatsache, daß auch in den größten Gemeinden die herrorragenden Kollegien einheitlich gestaltet sind und in der offiziellen Benennung den Namen der Kommune 
führen. $\mathrm{Ob}$ die Autorisierung durch die Munizipalgemeinde erfolgen kann oder notwendig von der Regierung erteilt werden muß, kommt hier weiter nicht in Betracht.

Vermögensrechtlich wird zunächst das conlegium ignoriert. Es führt keineswegs mit Notwendigkeit zu vermögensrechtlicher Gesammtaktion; insbesondere bei den Magistratskollegien weist keine Spur auf Bildung einer Sammtkasse, obwohl dazu zum Beispiel bei den Spielgeldern der Aedilen wohl Veranlassung gewesen wäre.

Sammtkassen dagegen haben die sakralen Kollegien wohl alle und seit alter Zeit gehabt - man erinnere sich nur an das sacramentum, überhaupt an die aus den staatlichen Fundationen und aus den Eintrittsgeldern ihnen zufließenden regelmäßigen Einnahmen, sowie an die für den Gottesdienst erforderlichen ständigen Ausgaben. Aber dies arcam habere gibt diesen Kollegien wohl eine faktische, aber keineswegs rechtliche Selbständigkeit; vermögensrechtlich bleibt das Rechtssubjekt auch hier zunächst der Staat, resp. die Stadt. DaB dies gerade bei den ältesten und höchsten dieser Kollegien auch praktisch stets festgehalten worden ist, zeigt die bekannte Tatsache, daß zum Beispiel der Pontificat und die Arvalen, obwohl sie eine eigene Kasse haben, über deren Forderungen und Verpflichtungen durch Kollegienbeschluß entschieden wird, dennoch keinen eigenen Sklaven besitzen können, sondern lediglich sich der in ihren Dienst gestellten Sklaven der Gemeinde bedienen. Es sind also dies öffentliche Kassen, von der Hauptkasse der Gemeinde insofern schärfer getrennt als die magistratischen, als sie nicht mit jener abrechnen und Überschüsse abliefern, aber keineswegs als eigene Rechtssubjekte dem Populus gegenübergestellt.

Aber bei den geringeren Kollegien dieser Art ist eine derartige Verselbständigung vorgenommen worden, für die als Kriterium wie auch wohl als praktischer Ausgangspunkt der Besitz eigener Sklaven betrachtet werden kann; die technische Bezeichnung dafür ist corpus habere. Offenbar erschien es nicht angemessen, dem Sklaven einer solchen untergeordneten Gemeinschaft die privilegierte Stellung des servus publicus einzuräumen und einer solchen arca die 
Stellung einer separat verwalteten Staatskasse zu geben. Es ist daher charakteristisch, daß die Quästoren sich der servi publici, die Dekurien der quästorischen Schreiber aber der eigenen Sklaven bedienen. Der Rechtsgrund für eine derartige exzeptionelle Stellung kann begrifflich nur in staatlicher Konzession gefunden werden, wenn dieselbe auch vielfach ohne eigentliche Sanktion auf dem Wege des gewohnheitsrechtlich zugelassenen Abusus konstituiert worden sein mag. Mit dem Assoziationsrecht hängt dieselbe zunächst nur insofern zusammen, als, wo dieses fehlt, natürlich auch sie nicht eintreten kann; keineswegs aber hat umgekehrt jedes anerkannte Kollegium auch corpus. Erst Kaiser Marcus hat beides insofern verknüpft, als er allen vom Staat anerkannten Kollegien das Korpus beilegté (Dig. 40, 3, 1 pr.).

Was den Umfang der in dem corpus enthaltenen Rechte anlangt, so ist die Ordnung desselben ad exemplum rei publicae dabei der leitende Gedanke. Auch hat es praktisch keine Schwierigkeit, dem Kollegium die vermögensrechtliche Handlungsfähigkeit in dem gleichen Sinne beizulegen, wie sie der Stadtgemeinde zusteht; die Gestion, welche dasselbe eben wie die Stadt administrativ ausübt, kann ohne weiteres auf das Gebiet des Vermögensrechtes übertragen werden; ja die durch die persona incerta hervorgerufenen Bedenken stehen hier nicht im Wege. Für einzelne Rechte ist folgendes zu bemerken.

Das Recht der Freilassung ist von dem Recht Sklaven zu haben wohl theoretisch, aber schwerlich praktisch zu trennen. Regelmäßig wird also wohl mit der Anerkennung eines Kollegiums als Korpus eine Anordnung verbunden gewesen sein, welche die Freilassung ihm möglich machte, sie also entweder an den Beschluß des Kollegiums knüpft oder einem Vertreter desselben die Freilassung durch Vindicta gestattet.

Den Vermögenserwerb von Todes wegen insoweit zu gestatten, als er mit der Freilassung rechtlich verbunden war, ist ohne $Z$ weifel lediglich als Konsequenz derselben behandelt worden (Dig. 40,3,2). Hinsichtlich des Legats und der Erbschaft scheint genau dasselbe gegolten zu haben, was von der Stadtgemeinde gilt. Daß dem Kollegium, 
welchem corpus beigelegt war, nicht damit zugleich der Erwerb der Legate verstattet war, kann aus Dig. 34, 5, 20 nicht gefolgert werden; hier ist vielmehr offenbar nur ausgesprochen, daß das allen collegia licita von Marcus beigelegte corpus das Recht Legate $\mathrm{zu}$ erwerben involviere. Andererseits wird der Erbschaftsantritt dem Kollegium nur nach Spezialprivilegium gestattet (Cod. Iust. 6, 24, 8), ohne Frage ad exemplum rei publicae.

Aber auch auf einem anderen Wege als auf dem eben bezeichneten der Verselbständigung einer speziellen öffentlichen Kasse ist das römische Recht dazu gelangt, ein nicht mit einer physischen Person zusammenfallendes Vermögenssubjekt zu konstituieren. Die Vergesellschaftung zu ökonomischen Zwecken führt dies bekanntlich im allgemeinen nicht herbei; es kann wohl faktisch eine Gesellschaftskasse (arca communis Dig. 17, 2, 82) gebildet werden, aber rechtlich ist für alle vermögensrechtlichen Beziehungen der Gesellschaft entweder einer der Gesellschafter der Träger oder nach Quoten mehrere oder alle, wovon bekanntlich das römische Zivilrecht die Konsequenzen mit einer praktisch kaum erträglichen Strenge zieht. Aber wenn dies Zivilrecht notwendig da außer Kraft tritt, wo der Bürger mit der Gemeinde kontrahiert, ist hinsichtlich der Gesellschaft dies so weit ausgedehnt worden, dah, wo die Gesellschaft mit dem Staat kontrahiert, selbst die Verhältnisse der Gesellschafter zueinander dadurch alteriert werden und, um es mit einem Worte zu sagen, soweit das Interesse des Staates dies erfordert, der Gesellschaft corpus beigelegt wird, sie durch das Ausscheiden der Genossen nicht oder doch nicht notwendig aufgelöst wird und sie eigene Sklaven haben kann, welche so wenig wie die des Munizipiums von den Gesellschaftern nach Quoten besessen werden. Daß die Beilegung der Persönlichkeit durch die bloße Tatsache des Kontrahierens mit dem Staat herbeigeführt worden sei, ist nicht wahrscheinlich; wenn auch das Herkommen hier eingegriffen haben mag, führt doch auch die Ausdrucksweise der Juristen (Dig. 3, 4, 1 pr.) darauf, daß prinzipiell eine diesfällige Anerkennung durch die Gemeinde erforderlich war. Im Ergebnis fallen das Kollegium und die societas, insoweit beide corpus 
erhalten, wohl nur partiell zusammen; der Sache nach wird jenes nach dem Muster der Gemeinde zu behandeln sein, dieses aber nicht aufgehört haben, als societas zu gelten. Schulden des Kollegiums also wird man nicht gegen das einzelne Mitglied haben geltend machen können, wohl aber für Schulden auch der publizistischen Sozietät der socius nach den Regeln des Privatrechtes gehaftet haben. Bei Auflösung beider wird das Vermögen des Kollegiums als separiertes Staatsgut an die Gemeinde gefallen, das der Societas unter die Genossen verteilt worden sein.

\section{III. \\ Sanctio pragmatica. \\ Von \\ Theodor Mommsen.}

Aus dem Nachlasse des Verfassers herausgegeben.1)

Die hinsichtlich des Begriffs der pragmatica sanctio bestehenden Schwankungen und Irrtümer haben mich bestimmt, den Begriff derselben zu erörtern.

Pragmaticum rescriptum oder pragmatica sanctio, auch elliptisch pragmaticum oder pragmatica ${ }^{2}$ ), begegnet in techni-

1) S. die Redaktionsbemerkung S. 33 [Anm. d. Red.]. - ${ }^{2}$ ) Pragmaticum rescriptum findet sich im Donatistenprozeß vom J. 411 (coll. III, Carthag. Mansi vol. 4 p. 188 c. 38 ) und ebenso bei Augustinus (coll. III cum Donatistis c. 2 vol. 9 p. 553 Maur.). In dem auf Grund des praeceptum, quo (imperator) collationem iussisset fieri, [eingeleiteten Verfahren] exbitten die Donatisten von dem dadurch eingesetzten Richter zunächst die Verlesung des betreffenden Reskripts, was geschieht, dann auch die ihrer eine solche Verhandlung erbittenden Eingabe an den Kaiser, was der Cognitor abschlägt: pragmaticis rescriptis preces inseri non solere. - Pragmatica sanctio ist häufig; pragmatica iussio bes. in Nov. Theod. $17,1,3.17,2,5=$ Iust. $10,12,2$; pragmatica forma Iust. $5,5,9$,

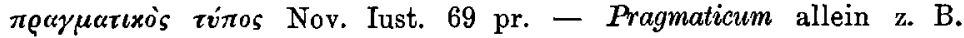
Theod. 6, 23, 3. 11, 1, 36. - Pragmatica allein z. B. Nov. Theod. 8 pr. 